Int. J. Speleol. 6 (1974), pp. 193-215.

\title{
Pseudoscorpions cavernicoles de la Macédoine
}

\author{
par \\ B. P. M. ĆURČIĆ*
}

SUMMARY

\section{Cavernicole Pseudoscorpions from Macedonia}

A cavernicole pseudoscorpion of the genus Neobisium Chamberlin 1930 is living in Kalina Dupka cave in the Bistra Highland of western Macedonia. This pseudoscorpion clearly differs from the other members of the subgenus Blothrus Schiфdte 1849, and belongs to the new species $N$. (B.) princeps, the principal features of which are described in this study. The nearest relatives of this species are $N$. (B.) spelaeum (Schiфdte) 1849, and $N$. (B.) stygium Beier 1931, both from Slovenian and Croatian caves. From chelal dentition $N$. (B.) princeps may be considered as the most primitive element of the princeps-stygium-spelaeum series. This new species is in a subterranean mode of life of extreme specialization. Relating to biogeography, it belongs to the endemic pseudoscorpion fauna in Macedonia.

After finding pseudoscorpions in Zmejovica cave (Poreče mountainous area), we confirmed the presence of the species N. (B.) karamani (Hadži) 1929 in west Macedonia. Morphologic analysis of male specimens from that new locality enabled us to complete description of this species previously based on a single female specimen. The comparison of Hadži's species with $N$. (B.) remyi Beier 1939 from west Serbian caves, with $N$. (B.) brevipes (Frivaldsky) 1866 and $N$. (B.) leruthi Beier 1931 from Turda and Bihar caves in southern Carpathians, leads to the conclusion that these pseudoscorpions belong to a closely related species group. Judging by actual distribution of these species, the possibility exists that a wide area in the ancient Balkanic dry land had been populated by the initial form of that series.

As for its preferences for habitat $N$. (B.) karamani is an exclusive inhabitant of subterranean environment. Relating to biogeography, it may be considered as a relic of mediterranean Tertiary fauna and its endemic differentiation as developed under the conditions of the evolution of karst relief in southern countries of the Balkan Peninsula.

In conclusion, from actual knowledge and the results of this study caves in Macedonia are inhabited by three endemic species of pseudoscorpions of genus Neobisium (Blothrus), namely: N. (B.) ohridanum Hadži 1940,N. (B.) karamani (Hadži) 1929, and N. (B.) princeps Ćurčić 1974. Judging by known blothroid pseudoscorpions, it is possible Macedonia represents one of the centres of origin and genesis for authochthonous and residual fauna of the Tertiary age.

Le sous-genre Blothrus Schiødte 1849 - du genre Neobisium Chamberlin 1930 habite l'Europe méridionale, depuis la Péninsule ibérique à l'Occident jusqu'à l'Asie Mineure à l'Orient. La limite septentrionale de l'aire de répartition de ces Pseudoscorpions embrasse la région de la Styrie du Nord-Ouest ("Nördliche Kalkalpen") et le territoire du Bihar dans les Alpes de Transylvanie. Les localités les plus méridionales de ce sous-genre sont celles de l'ile de Crète.

Dans la Péninsule des Balkans vivent trente-trois espèces de Pseudoscorpions

*) Institut za zoologiju, Prirodno-matematički fakultet, Studentski trg 16, 11000 Beograd, Yugoslavia. 
blothroïdes (Ćurčić, 1974; c'est pourquoi cette région est considérée comme un centre important de distribution du sous-genre Blothrus. Parmi toutes les espèces balkaniques, 26 habitent la partie occidentale de la Péninsule et en particulier les grottes du karst dinarique. Cependant, dans les parties centrale, orientale, et méridionale de la Péninsule, on n'a constaté jusqu'à présent que sept espèces de ce sousgenre. Ainsi, dans les grottes de la Serbie vivent le Neobisium (Blothrus) remyi Beier 1939, et le $N$. (B.) stankovici Ćurčić 1972, tandis que dans les grottes de la Bulgarie habite le $N$. (B.) kwartirnikovi Mahnert 1972. Dans les localités souterraines de l'Albanie on a récolté le $N$. (B.) albanicum (Müller) 1931, tandis qu'à Corfou on a trouvé le $N$. (B.) odysseum (Beier) 1929.

Dans les grottes de la Macédoine, d'après les connaissances actuelles, vivent deux espèces de Pseudoscorpions blothroïdes. L'une d'elles, le N. (B.) karamani (Hadži) 1929 a été décrite d'après le matériau récolté dans la grotte proche du village Patǐ̌ka aux environs de Skoplje. L'autre, le N. (B.) ohridanum Hadži 1940, habite la grotte Mečkina Dupka près de la ville d'Ohrid.

S'appuyant sur le fait que certaines espèces de Blothrus ont été trouvées dans les régions limitrophes de la Macédoine, Curčić (1972) pense qu'il est probable que ce sous-genre soit non seulement largement répandu dans les parties centrale et orientale de la Péninsule des Balkans, mais aussi dans sa partie méridionale. Cette opinion est confirmée par de nouvelles trouvailles de Pseudoscorpions blothroïdes, dont les principaux caractères morphologiques sont exposés dans le présent travail. Les échantillons de ces Pseudoscorpions ont été récoltés dans les grottes de la Macédoine occidentale; par l'identification taxonomique, il est établi que les exemplaires de la grotte Kalina Dupka appartiennent à la nouvelle espèce: $N$. (B.) princeps, tandis que les exemplaires de la grotte Zmejovica appartiennent à l'espèce $N$. (B.) karamani.

\section{NEOBISIUM (BLOTHRUS) PRINCEPS N. SP.* (Fig. 1-12)}

LOCALITE-TYPE - La grotte Kalina Dupka, à $3 \mathrm{~km}$ au Nord du village Lazaropolje et à $14 \mathrm{~km}$ (à vol d'oiseau) à l'Est de Debar, Macédoine occidentale; VIII-1953, S. Karaman leg.

La grotte Kalina Dupka appartient aux éléments du relief karstique souterrain de la montagne Bistra et s'est individualisée dans le massif calcaire du mont Sokolica. L'entrée de la grotte se trouve à une altitude d'environ $1200 \mathrm{~m}$ au-dessus du niveau de la mer (Josifovski, manuscrit).

Les galeries de la grotte Kalina Dupka sont remplis de blocs de pierre, recouverts partiellement d'argile humide. Au point de vue hydrographique, l'un des couloirs de la grotte est encore toujours actif; un cours d'eau souterrain, qui prend sa source au voisinage immédiat de la grotte sous le nom de Palav Izvor, coule le long de ce

\footnotetext{
*) DERIVATION DU NOM - Lat.: princeps = le premier du genre; en fonction des caractères qui la distinguent de toutes les autres espèces du genre. Le $N$. (B.) princeps se distingue de toutes les autres espèces connues actuellement par l'extrème gracilité de ses pattes-mâchoires et le grand nombre de dents aux doigts de la pince.
} 
passage souterrain. Au point de vue géomorphologique, le système des galeries de la grotte Kalina Dupka s'est formé par l'action combinée de l'eau du cours souterrain et des eaux de suintement.

\section{Adulte (femelle)}

La chitine de la carapace et de l'abdomen est jaunâtre et transparente. Les chélicères sont jaunâtres, tandis que les pattes-mâchoires sont de nuance brunrougeâtre; seules les parties fortement chitinisées sont de couleur plus sombre (régions articulaires). Les articles des pattes marcheuses sont presque incolores et transparents.

CEPHALOTHORAX - La carapace a la forme d'un rectangle presque régulier; elle est notablement plus longue que large ( 1.40 fois). Elle atteint sa plus grande largeur au niveau de la rangée des soies 'oculaires'. L'épistome n'est pas développé; la partie centrale du bord antérieur du céphalothorax est légèrement convexe, arrondie et lisse.

Les yeux ne sont pas développés; il n'y a pas trace non plus de taches oculaires. Il n'y a que 19 (paratype) ou 21 (holotype) de soies céphalothoraciques. Ainsi, le bord antérieur du céphalothorax porte 4 soies; dans la série 'oculaire' il y a 6 (holotype) ou 7 soies (paratype). Dans les séries médiane et intermédiaire, les soies peuvent être disposées irrégulièrement (holotype), ou par 4 dans chacune des séries citées (paratype). Le bord postérieur du céphalothorax porte 3 (holotype) ou 4 soies (paratype).

ABDOMEN - Les plaques tergales sont lisses, tandis que les pleures portent des granulations. Le nombre de soies à chaque tergite n'est pas le même dans les deux exemplaires. Ainsi, le tergite I porte 4 ou 6 soies, le tergite II -4 , le tergite III -6 , et le tergite IV -5 ou 6 soies. Les deux tergites suivants, le V et le VI, sont munis chacun de 6 ou 7 soies. Le tergite VII présente 7 ou 8 soies, et le tergite VIII -7 ou 9 soies. Le tergite IX porte 8 ou 10 soies, et le tergite X -8 ou 9 soies. Ainsi, la chétotaxie de la partie dorsale de l'abdomen est, dans le holotype, 6-4-6-5-6-7-7-7-8-8, et dans le paratype: 4-4-6-6-7-6-8-9-10-9. Les soies sont de longueur différente; à partir du quatrième tergite vers la partie postérieure de l'abdomen, le paire de soies latérales devient plus longue par rapport aux auttes soies du même tergite.

L'opercule génital porte 13 (holotype) ou 15 soies (paratype). La plaque génitale postérieure présente une rangée de 12 (holotype) ou 14 soies (paratype). Ce sternite porte encore 3 microchètes suprastigmatiques de chaque côté.

La papille anale porte 2 paires de soies.

CHELICERES - Les sveltes chélicères sont 2.09 fois plus longues que larges. Le tubercule fileur est petit et aplati, mais bien évident. La lame du doigt mobile porte, distalement, cinq petites dents obtuses, de forme irrégulière. Derrière ces dents vient la partie surélevée de la lame, dont le bord est irrégulièrement denté (il est possible que cette partie de la lame corresponde à une grande dent à la longue usée et rendue obtuse). La lame du doigt fixe porte distalement de 8 à 10 petites dents serrées les unes contre les autres, de forme irrégulière et de taille croissante vers le 
milieu du doigt. A la suite de ces dents vient une série de 9 ou 10 dents plus grandes, triangulaires et espacées les unes des autres.

La main porte 6 soies (mais la main de la chélicère gauche de l'holotype présente 5 soies), tandis que le doigt mobile n'en possède qu'une. Le flagelle est constitué par 8 soies de longueur inégale; les deux plus longues sont pennées d'un côté. La grandeur des autres soies flagellaires diminue vers l'extrémité proximale. La serrule externe présente 36 ou 38 lames, la serrule interne -35 .

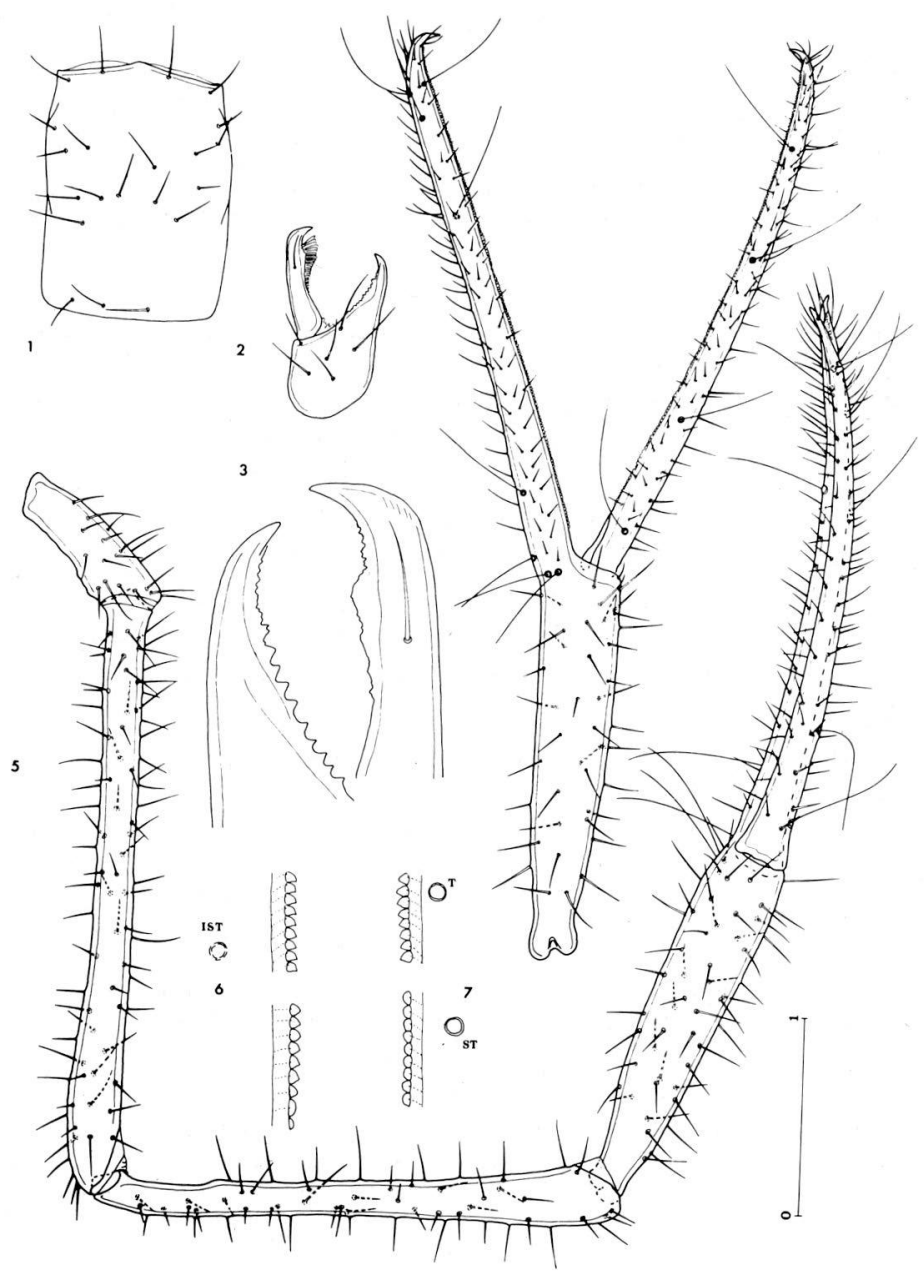

Fig. 1-7. Neobisium (Blothrus) princeps n. sp., femelle-holotype. (1) carapace; (2) chélicère; (3) doigts de la chélicère; (4) pince; (5) patte-mâchoire; (6) dents du doigt fixe de la pince (au niveau de la trichobothrie IST et à la base de la lame); (7) dents du doigt mobile de la pince (au niveau des trichobothries T et ST). Echelle en $\mathrm{mm}$. 
PATTES-MACHOIRES - Les pattes-mâchoires sont très sveltes et presque trois fois plus longues que le corps. Tous les articles de cette paire d'appendices sont lisses ainsi que très allongés. Sur le lobe maxillaire de la hanche se dressent 5 longues soies, tandis que la coxa porte 6 soies. Le trochanter porte un très petit tubercule.

Le fémur et le tibia sont droits; ces deux articles présentent la plus grande largeur dans leur partie distale. La main de la pince présente des bords presque parallèles. Latéralement observée, la main est la plus large dans la région de la base des doigts et s'amincit vers le pédoncule.

Les doigts de la pince sont longs ( 1.44 fois plus longs que la main), droits et munis d'un grand nombre de dents. Les extrémités des doigts sont recourbées en crochet et allongées. Le doigt fixe porte en tout 176 (holotype) ou 177 (paratype) dents serrées et à peu près de même taille. Dans la moitié distale de la lame, ces dents sont sensiblement de même longueur et de même largeur et en plus asymétriques; dans la partie distale (= terminale) de la couronne, chacune de ces dents porte une petite cupule d'émail. Ce n'est qu'à l'extrémité proximale de la lame du doigt fixe que les dents sont plus larges que longues et, en même temps, arrondies.

La lame du doigt mobile porte 153 (paratype) ou 155 (holotype) dents, n'atteignant pas le niveau de la trichobothrie B. Dans le quart distal de la lame, les dents sont asymétriques, serrées les unes contre les autres, aussi longues que larges. Leurs sommets portent des cupules d'émail. A partir du niveau de la trichobothrie T, la longueur (= hauteur) des couronnes dentaires diminue; celles-ci deviennent plus arrondies, de telle sorte que la lame se termine par des dents obtuses, larges et basses.

A la base du côté interne de la lame, le doigt mobile possède une dépression aux bords chitinisés. Sur le côté latéral externe, ce doigt porte 4 trichobothries. Le doigt fixe a 8 trichobothries, dont 4 situées près de la base du doigt.

PATTES AMBULATOIRES - La hache de la première paire de pattes marcheuses présente un processus antéro-latéral conique, dirigé vers l'extrémité antérieure. La partie distale de ce processus est incolore et transparente, tandis que sa base est de couleur chatain. L'angle antérieur médian de la coxa I est arrondi et porte de 2 à 4 très petites pointes. La région antéro-latérale de la coxa II est de couleur chatainrougeâtre.

La coxa I porte 4 soies, la coxa II - 5 ou 6 , la coxa III - 4 ou 5 , et la coxa IV 8 ou 9 soies.

Les poils subterminaux du télotarse IV sont biramés; chez les exemplaires analysés ces poils sont de forme différente.

DIMENSIONS (EN MM) - v. Tableaux $1 \& 2$.

MATERIAU - Une femelle-holotype et une femelle-paratype. 

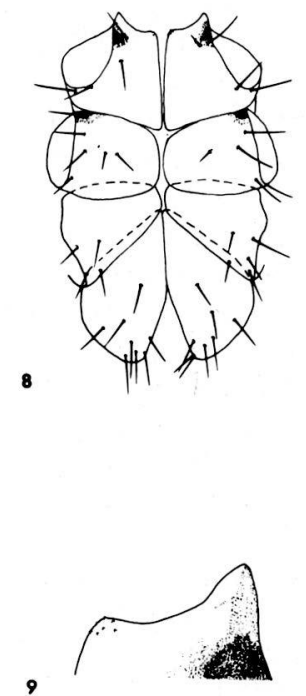

10

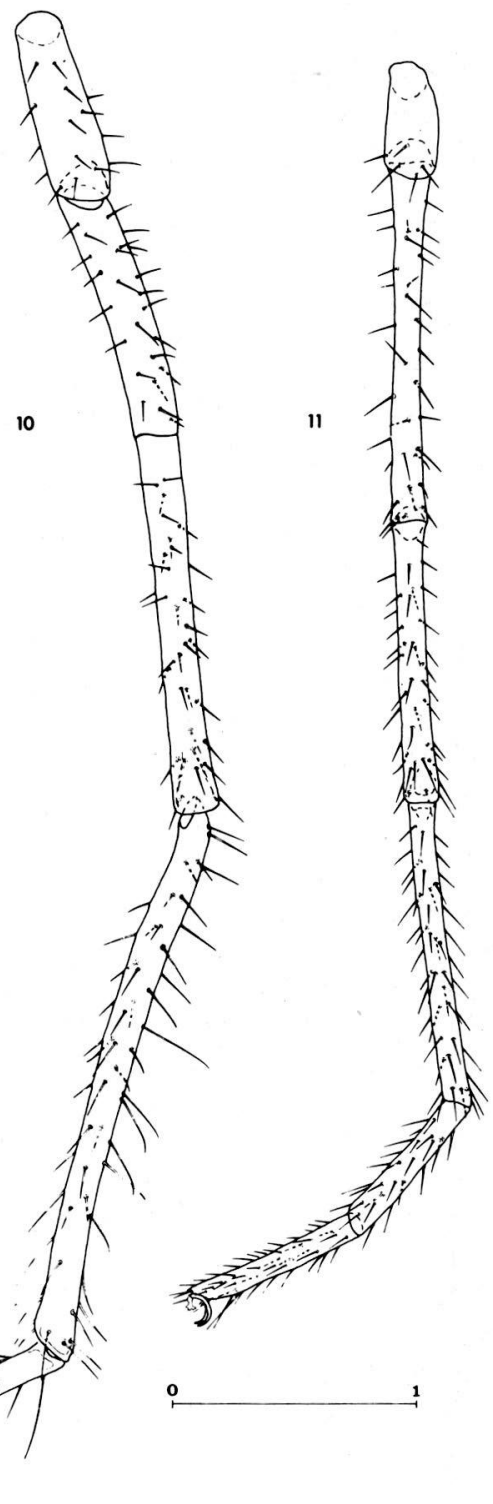

Fig. 8-12. Neobisium (Blothrus) princeps n. sp., femelle-holotype. (8) région coxale; (9) bord antérieur de la coxa I; (10) patte ambulatoire IV; (11) patte ambulatoire II; (12) poil subterminal du télotarse IV. Echelle en $\mathrm{mm}$. 
Tableau 1. Les dimensions (en $\mathrm{mm}$ ) et les rapports morphométriques des structures différentes chez le Neobisium (B.) princeps n. sp.

Corps

caractère

femelle-holotype

1. longueur

4.42

Céphalothorax

2. longueur

1.30

3. longueur du bord antérieur

0.84

4. largeur maximale

0.93

5. rapport: $2 / 4$

1.40

6 . longueur du bord postérieur

0.89

7. rapport: $3 / 6$

0.94

Abdomen

8. longueur

3.12

9. largeur

Chélicères

10. longueur

11. largeur

0.425

12. épaisseur

0.35

13. longueur du doigt mobile

0.57

14. rapport: $10 / 13$

1.56

Pattes-mâchoires

15. longueur (avec la hanche)

12.565

16. longueur (sans la hanche)

11.575

17. rapport: $15 / 1$

2.84

18. longueur de la hanche

0.99

19. longueur du trochanter

0.93

20. largeur du trochanter

0.31

21. longueur du fémur

3.05

22. largeur minimale du fémur

0.185

23. largeur maximale du fémur

0.27

24. rapport: $21 / 23$

11.30

25 . longueur du tibia

2.76

26. largeur du tibia

0.32

27. rapport: $25 / 26$

8.625

28. longueur de la pince

4.835

29. largeur de la pince

0.41

30. rapport: $28 / 29$

11.79

31 . longueur de la main

32. rapport: $31 / 29$

4.77

33. longueur du doigt mobile

34. longueur du doigt fixe

2.88

35. rapport: $34 / 31$

1.44

36. distance de la base du doigt à $\mathrm{B}$

0.25

37. distance de $\mathrm{B}$ à SB

0.60

38. distance de SB à ST

0.87

39. distance de ST à T

0.58

40. distance de $\mathrm{T}$ au sommet du doigt

0.49 
Tableau 2. Les dimensions (en $\mathrm{mm}$ ) et les rapports morphométriques des structures différentes chez le Neobisium (B.) princeps sp. n.

caractère femelle-holotype

Patte ambulatoire II

1. longueur (avec la hanche)

6.71

2. longueur (sans la hanche)

6.23

3. longueur de la hanche

0.48

4. largeur de la hanche

0.36

5. longueur du trochanter

0.48

6. largeur du trochanter

0.25

7. longueur du fémur I

1.58

8. largeur du fémur I

0.20

9. longueur du fémur II

1.18

10. largeur du fémur II

0.15

11. longueur du tibia

1.28

12. largeur du tibia

0.12

13. longueur du basitarse

0.74

14. largeur du basitarse

0.12

15. longueur du télotarse

0.97

16. largeur du télotarse

0.11

Patte ambulatoire IV

17. longueur (avec la hanche)

18. longueur (sans la hanche)

19. longueur de la hanche

0.65

20. largeur de la hanche

0.34

21. longueur du trochanter

0.82

22. largeur du trochanter

0.26

23. longueur du fémur

2.71

24. largeur du fémur

0.20

25. longueur du tibia

2.455

26. largeur du tibia

0.16

27. longueur du basitarse

0.93

28. largeur du basitarse

0.14

29. longueur du télotarse

1.35

30. largeur du télotarse

0.12

Les relations du Neobisium (B.) princeps avec les autres espèces du genre

La nouvelle espèce diffère notablement de tous les représentants du genre Neobisium (sous-genre Blothrus) connus jusqu'à présent. En même temps, elle montre différents degrés de ressemblances avec certaines espèces des localités souterraines de la Péninsule des Balkans et de l'Asie Mineure. 
Comparée au $N$. (B.) albanicum de la grotte Vrima e dragoi, la seule espèce blothroïde albanaise connue jusqu'à présent, l'espèce $N$. (B.) princeps se distingue nettement par l'absence d'épistome, par la longueur relativement plus grande de la carapace, par le nombre plus petit de soies tergales, par quelques rapports morphométriques (dans la nouvelle espèce, par exemple, les doigts de la pince sont plus courts, et dans l'espèce albanicum plus longs que le fémur de la patte-mâchoire), et par la longueur relative et absolue des articles de la patte-mâchoire. Mais à côté de ces différences, les deux espèces analysées présentent des ressemblances dans la dentition du doigt mobile des chélicères et, dans une certaine mesure, dans celle des doigts de la pince.

De l'espèce $N$. (B.) stankovici de la grotte Velika Pećina en Serbie orientale et de l'espèce voisine de celle-ci, le $N$. (B.) kwartirnikovi de la grotte Duhlata en Bulgarie, la nouvelle espèce diffère par l'absence d'épistome, par des appendices absolument et relativement plus longs et par la longueur plus grande du corps. Des différences beaucoup plus nettes concernent la forme des articles de la patte-mâchoire (dans l'espèce princeps, ils sont plus sveltes que dans l'espèce stankovici), le rapport des longueurs de la main et des doigts de la pince ( 1 : 1.44 chez le princeps, $1: 1.17$ $1.30 \mathrm{chez}$ le stankovici, et $1: 1.16 \mathrm{chez}$ le kwartirnikovi), et la dentition de la pince (la nouvelle espèce possède $176 \mathrm{ou} 177$ dents au doigt fixe et de 153 à 155 dents au doigt mobile, dans les espèces stankovici et kwartirnikovi le nombre des dents au doigt fixe s'élève à 139 et au doigt mobile à 112). Des distinctions entre les trois espèces que nous comparons s'observent également dans de nombreux rapports morphométriques (par ex.: le rapport de la longueur à la largeur du fémur des pattes-mâchoires dans l'espèce stankovici varie de 6.96 à 7.14, dans l'espèce kwartirnikovi il est 8.20, et dans la nouvelle espèce il atteint même 11.30! ).

Cependant, les espèces princeps, stankovici et kwartirnikovi présentent aussi certaines ressemblances ayant trait à la chétotaxie de la carapace et de la partie dorsale de l'abdomen, ainsi qu'à la dentition du doigt mobile de la chélicère.

Par rapport au N. (B.) sbordonii Beier 1973, qui habite la grotte Guezeu près de Maraș en Anatolie, la nouvelle espèce est caractérisée par un plus petit nombre de soies céphalothoraciques, par les articles des pattes-mâchoires relativement et absolument plus longs, et par la forme différente du tibia et de la main des pattesmâchoires (dans la nouvelle espèce, le tibia est notablement moins élargi dans sa partie distale que dans l'espèce sbordonii; de même, la main du princeps a des bords paralleles, tandis que chez le sbordonii la main est ovale); des différences entre ces deux espèces se remarquent aussi dans certains rapports morphométriques (par ex.: dans l'espèce princeps, les doigts de la pince sont plus courts, tandis que dans l'espèce sbordonii plus longs que les fémurs des pattes-mâchoires).

Cependant, les analogies entre ces deux espèces concernent la chétotaxie de la partie dorsale de l'abdomen, la chétotaxie des chélicères, et quelques rapports morphométriques.

En comparant les exemplaires de la nouvelle espèce avec des exemplaires typiques de deux espèces macédoniennes connues jusqu'à présent, on constate aussi de différences notables. Ainsi, le $N$. (B.) princeps diffère du N. (B.) ohridanum par l'absence de taches oculaires, par l'absence d'épistome et par un plus petit nombre 
de soies au bord postérieur de la carapace. En outre, les deux espèces considérées se distinguent par la chétotaxie du côté dorsal de l'abdomen et de la main des chélicères, par la forme et la longueur relative et absolue des articles des pattesmâchoires, ainsi que par la dentition des doigts de la pince. Mais, à côté de ces différences, les espèces princeps et ohridanum présentent des dentitions semblables aux chélicères et, dans une certaine mesure, une même forme de dents aux doigts de la pince.

La seconde espèce macédonienne, le $N$. (B.) karamani s'éloigne aussi de la nouvelle espèce par toute une série de caractères distinctifs; par la comparaison des exemplaires typiques de ces deux espèces, on constate que la nouvelle espèce a un corps et des appendices plus longs, qu'elle ne possède pas d'épistome et que les articles de ses pattes-mâchoires présentent une forme différente de celle des mêmes articles dans l'espèce karamani (par ex.: le tibia de l'espèce princeps est presque droit, tandis que celui de l'espèce karamani est en massue et courbe; ensuite, la main de la nouvelle espèce présente des bords parallèles, tandis que celle de l'espèce karamani est ovale). De même, dans la nouvelle espèce les doigts de la pince sont droits et de longueur égale, contrairement à ceux de l'espèce karamani, qui sont inégaux et sigmoïdes, en sorte qu'ils se croisent lorsque la pince se ferme. Ces deux espèces comparées présentent aussi des différences quant à la forme et au nombre de dents aux doigts fixe et mobile de la pince.

A côté de ces distinctions, les espèces princeps et karamani présentent certaines similitudes en fonction desquelles on peut admettre que ces deux espèces sont, du point de vue taxonomique, plus proches que ne le sont les espèces princeps et ohridanum. Ces similitudes concernent la longueur relative de la carapace, la chétotaxie de la carapace et du côté dorsal de l'abdomen, la forme, la chétotaxie et la dentition des chélicères, ainsi que la forme des doigts de la pince.

Parmi tous les représentants du sous-genre Blothrus, la nouvelle espèce se rapproche le plus de l'espèce $N$. (B.) stygium Beier 1931 (= minoum Beier 1931) habitant les grottes de la Croatie et du Sud-Est de la Slovénie, et de l'espèce $N$. (B.) spelaeum (Schiødte) 1849, trouvée dans les localités souterraines de la Slovénie et de l'Istrie.

Par l'analyse comparée des exemplaires typiques et autres de l'espèce stygium et de ceux de la nouvelle espèce, on constate que l'espèce princeps se distingue par des articles de pattes-mâchoires plus graciles et relativement plus longs, par la forme différente de la main (dont les bords sont parallèles, contrairement à la main ovale de l'espèce stygium), par la forme différente des doigts de la pince (dans la nouvelle espèce ces doigts sont droits et chez le stygium ils sont légèrement sigmoïdes). En outre, la nouvelle espèce se distingue aussi par un plus grand nombre de dents à la pince (176-177 au doigt fixe, et 153-155 au doigt mobile) que dans l'espèce stygium (79-90 dents au doigt fixe et 73-85 dents au doigt mobile), et par leur forme, leur disposition et leur taille différente (dans la partie distale de la lame de tous les deux doigts de la nouvelle espèce, les dents sont petites, asymétriques et serrées les unes contre les autres, tandis que dans l'espèce stygium elles sont grandes, symétriques, et espacées les unes des autres). L'espèce princeps diffère de l'espèce stygium par un plus grand développement du processus conique antéro-latéral des coxas de la première paire des pattes marcheuses. 
En comparant des exemplaires de l'espèce spelaeum, provenant de la localitétype et d'autres stations, avec des exemplaires de l'espèce princeps, on remarque des différences se rapportant à la longueur relative des articles des pattes-mâchoires (la nouvelle espèce a des pattes-mâchoires plus graciles), à la forme du tibia de la patte-mâchoire (le tibia de la nouvelle espèce est distalement moins élargi), à la forme de la main (chez le princeps, la main a la plus grande largeur à la base des doigts, tandis que chez le spelaeum elle est la plus large vers son milieu). En outre, le nombre des dents de la pince dans l'espèce princeps est notablement plus grand (176-177 au doigt fixe et 153-155 au doigt mobile contre 79-85 au doigt fixe et 65-78 au doigt mobile dans l'espèce spelaeum), leurs dimensions sont plus petites, et leur disposition et forme dans la partie distale de la lame des deux doigts sont différentes (dans la nouvelle espèce macédonienne, les dents sont plus petites, serrées les unes contre les autres et asymétriques, tandis que dans l'espèce spelaeum, elles sont plus grandes, éloignées les unes des autres, et symétriques - triangulaires).

Les trois espèces analysées: princeps, stygium et spelaeum se rapprochent l'une de l'autre par de nombreuses analogies: haut degré de réduction de l'épistome, chétotaxie de la carapace et de l'abdomen, dentition des chélicères, dentition des parties proximales de la pince, ainsi que certains rapports morphométriques.

D'après l'homodentition de la pince et d'après le très grand nombre de dents à la pince, il est probable que le $N$. (B.) princeps soit l'élément le plus primitif de la série princeps-stygium-spelaeum. A en juger d'après la répartition actuelle des espèces de la dite série, on peut supposer que la forme initiale de cette série ait été largement répandue dans les anciennes terres balkaniques.

A notre avis, le $N$. (B.) princeps est un troglobie prononcé, spécialisé à l'extrême à la vie cavernicole. De nombreuses adaptations nous le confirment: absence du pigment et des yeux, gracilité et longueur des appendices, petit nombre de soies aux coxas des pattes ambulatoires, etc. En outre, le degré d'adaptation de cette espèce aux conditions cavernicoles indique qu'elle a dû peupler les stations souterraines il y a bien longtemps et en tout cas avant les espèces cavernicoles dont les stades subadultes sont encore toujours caractérisés par la présence de petites taches oculaires et par des pattes-mâchoires 'robustes', comme c'est le cas avec le $N$. (B.) ohridanum. Etant donné que le $N$. (B.) princeps n'a été jusqu'à présent trouvé que dans une seule grotte en Macédoine occidentale, il est possible d'affirmer que cette espèce appartient à la faune des Pseudoscorpions endémiques sud-balkaniques, et qu'elle est très probablement exclusivement macédonienne.

\section{NEOBISIUM (BLOTHRUS) KARAMANI (HADŽI) 1929 (Fig. 13-35)}

En 1929, Hadži a décrit cette espèce d'après un seul exemplaire femelle, en faisant la remarque que malgré des recherches ultérieures dans la même localité, il n'avait pas réussi à trouver d'autres exemplaires. Ce n'est qu'au bout de 40 ans, grâce à la récolte de Pseudoscorpions dans la grotte Zmejovica que nous pouvons confirmer la présence de cette espèce dans les grottes macédoniennes et, en même temps, compléter sa description d'après des exemplaires du sexe opposé. La nouvelle localité n'est éloignée, à vol d'oiseau, que de $40 \mathrm{~km}$. de la localite-type du $N$. (B.) karamani. 
LOCALITE - La grotte Zmejovica, $2.5 \mathrm{~km}$ à l'Est du village Krapa et $32 \mathrm{~km}$ à l'Est de Kičevo (en ligne droite), Macédoine occidentale; 24-I-1968, R. Lindeman leg.

La grotte Zmejovica s'est formée dans les calcaires constituant la bordure de la crête Savirupi (= Margare-Dolgavec); ce massif appartient au Poreče, région montagneuse de la partie centrale du bassin de la rivière Treska. L'entrée de la grotte se trouve à une altitude d'environ $1460 \mathrm{~m}$ (Jovanović 1928).

$\mathrm{Au}$ point de vue géomorphologique, la grotte Zmejovica est constituée d'une simple chambre souterraine, partagée en plusieurs parties par des stalagmites et stalactites. Le sol de la grotte est couvert par des blocs de pierres tombés du plafond. Etant donné que dans la grotte on ne peut remarquer les traces de l'activité érosive de cours d'eau souterrains de quelque importance, Jovanović (1928) pense qu'elle a du se former sous l'action de l'érosion karstique chimique.

\section{Adulte (mâle)}

Les parties du corps plus fortement chitinisées (la carapace, les chélicères, les pattesmâchoires et les régions articulaires des pattes marcheuses) sont de couleur chatainrougeâtre; la chitine de l'abdomen et des pattes ambulatoires est très tendre et de couleur jaune clair.

CEPHALOTHORAX - La carapace est de 1.22 à 1.26 fois plus longue que large, c'est-à-dire qu'elle est relativement plus courte que chez la femelle, L'épistome triangulaire est très distinct; sa base peut être plus longue ou plus courte que les côtés latéraux. Au sommet de l'épistome il y a de nombreuses petites pointes.

$\mathrm{Ni}$ les yeux, ni les taches oculaires ne sont développés. Il y a 20 ou 19 soies céphalothoraciques; elles sont disposées comme chez la femelle. Ainsi, la rangée antérieure est formée de 4 soies, la rangée 'oculaire' de 6 , la rangée médiano -intermédiaire de 6 (ou 5), tandis que la rangée postérieure n'a que 4 soies.

13

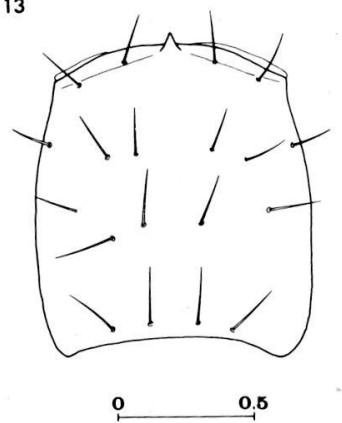

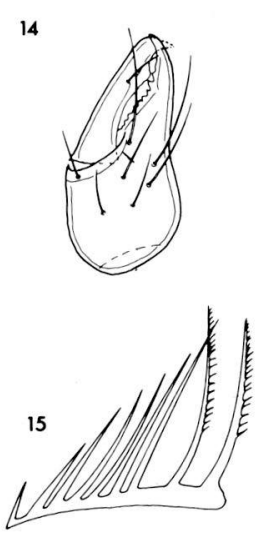

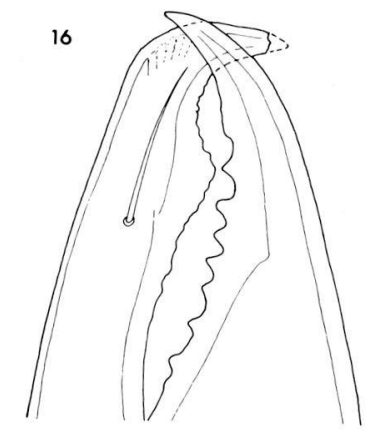

Fig. 13-16. Neobisium (Blothrus) karamani (Hadži) 1929, mâle $\mathrm{N}^{\mathrm{O}} 1$.

(13) carapace; (14) chélicère; (15) flagelle; (16) doigts de la chélicère. Echelle en $\mathrm{mm}$. 
ABDOMEN - La chétotaxie du côté dorsal de l'abdomen correspond à celle de la femelle. Ainsi, les tergites I et II portent 4 soies chacun; les deux tergites suivants sont munis de 6 soies chacun, tandis que les tergites V et VI portent 7 soies chacun; le tergite VII a 7 ou 8 soies, le tergite VIII -8 soies, le tergite IX -7 ou 8 soies, et le tergite $\mathrm{X}-8$ soies. Les formules tergales des exemplaires analysées sont: 4-4-6-6-7-7-8-8-8-8, et 4-4-6-6-7-7-7-8-7-8. Les soies latérales des tergites IV à X sont plus longues que les autres.

L'opercule génital porte 18 ou 21 soies; la plaque génitale postérieure est munie de 32 ou 33 soies. De ces soies, 17 appartiennent à la rangée transversale, tandis que 15 ou 16 soies forment un groupe dans la partie centrale du sternite. Le sternite III présente en plus 4 microchètes suprastigmatiques de chaque côté. Le sternite IV porte 11 ou 12 soies et 3 (4 dans un seul cas) microchètes au-dessus de chaque stigmate. Le sternite V possède 12 soies et le sternite VI - 15 ou 16 soies; les sternites VII-X portent 15 soies chacun.

La papille anale porte 2 paires de soies.

CHELICERES - Les chélicères sont plus de 2 (de 2.13 à 2.14) fois plus longues que larges. Cependant, elles sont relativement plus courtes que chez la femelle. Le tubercule fileur est presque complètement aplati. La dentition des chélicères est semblable à celle de la femelle. Ainsi, le doigt mobile présente, distalement, de 7 à 9 petites dents basses, arrondies et obtuses; derrière ces dents, la lame s'élève en une grande dent, échancrée à plusieurs reprises, et de forme irrégulière. Sur un exemplaire, les inégalites de la grande dent sont usées. La lame du doigt mobile se termine par 5 ou 6 petites dents triangulaires serrées les unes contre les autres, de taille inégale, ou bien par une série de 3 ou 4 dents basses et aplaties. Le doigt fixe porte, distalement, de 2 à 4 petites dents, obtuses et serrées les unes contre les autres, de taille inégale. A leur suite, vient une serie de 7 ou 8 grandes dents triangulaires, au sommet arrondies et espacées les unes des autres. Dans la moitié distale de la lame, entre les grandes dents, de petites dents intermédiaires peuvent être developpées.

La main de la chélicère porte 7 soies (il n'y a que la main d'un spécimen mâle qui porte 8 soies), tandis que le doigt mobile en porte une. Le flagelle est formé de 8 ou 9 soies; deux soies distales sont pennées d'un côté. Le nombre de lames des serrules est différent de celui chez la femelle. Ainsi, la serrule externe porte 37 ou 39 lames ( 45 chez la femelle), et la serrule interne n'a que 33 lames ( $30 \mathrm{chez}$ la femelle).

PATTES-MACHOIRES - Les graciles pattes-mâchoires sont plus de trois fois plus longues que le corps et semblables à celles de la femelle. Tous les articles de cette paire d'appendices sont lisses, sans granulations. Le lobe maxillaire porte un faisceau de 5 soies, tandis que la coxa porte 6 soies. Sur le trochanter, on note la présence d'un très petit tubercule aplani.

Le fémur est droit et élargi dans sa partie distale. Le tibia en forme de massue est élargi dans sa partie distale. La main de la pince est ovale et présente la plus grande largeur dans sa partie moyenne. Les doigts de la pince sont de 1.52 à 1.65 fois aussi longs que la main. Ils sont de longueur différente, de forme sigmoïde et ils se croisent lorsque la pince se ferme. Les extrémités des doigts sont crochues et 


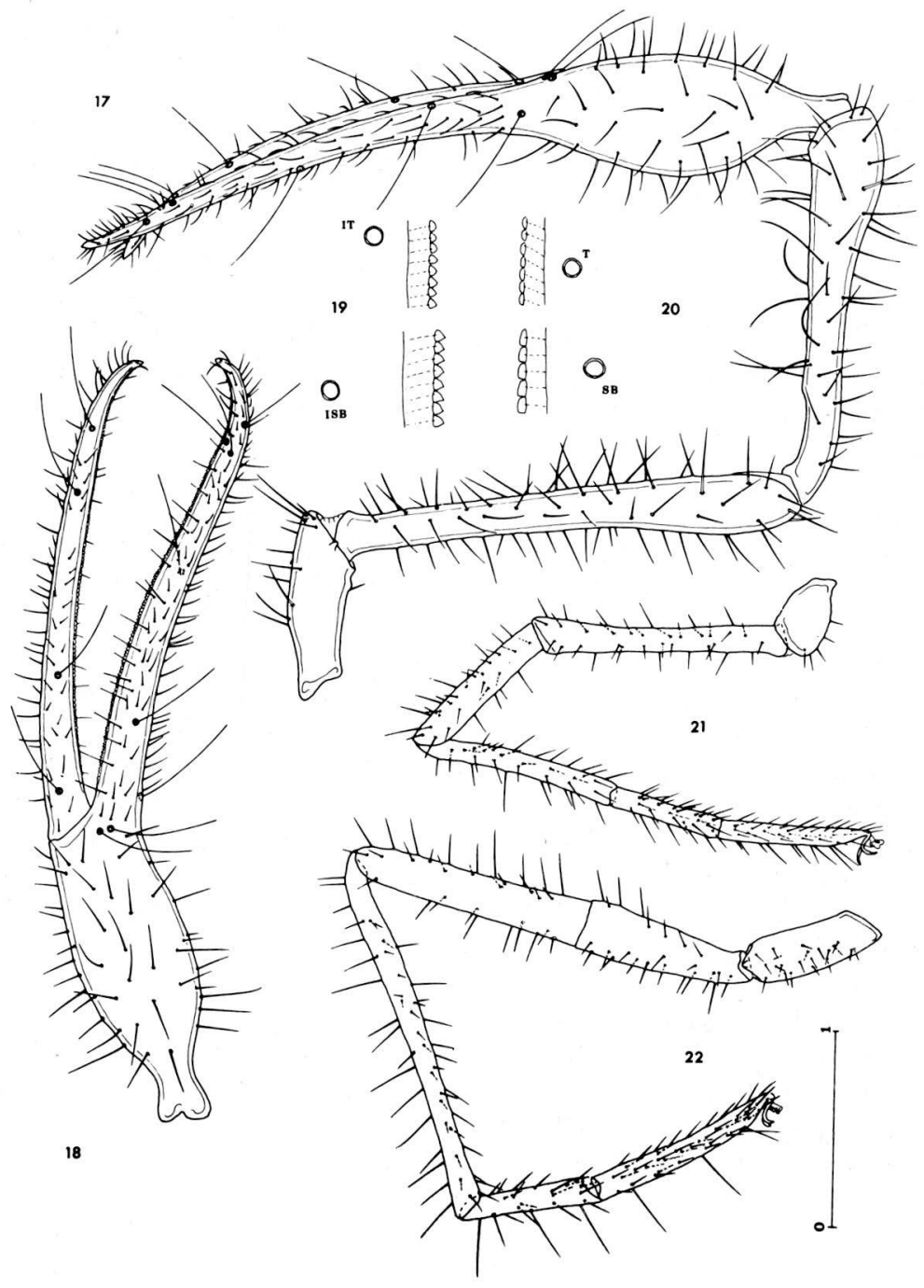

Fig. 17-22. Neobisium (Blothrus) karamani (Hadži) 1929, mâle $\mathrm{N}^{\mathrm{O}} 1$.

(17) patte-mâchoire; (18) pince; (19) dents du doigt fixe de la pince (au niveau des trichobothries IT et ISB); (20) dents du doigt mobile de la pince (au niveau des trichobothries T et SB); (21) patte ambulatoire II; (22) patte ambulatoire IV. Echelle en $\mathrm{mm}$. 
23

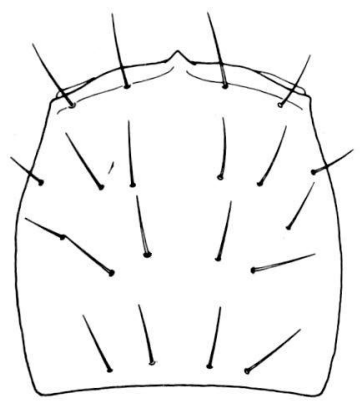

0

24

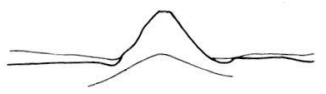

25

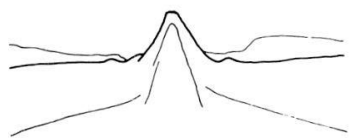

26
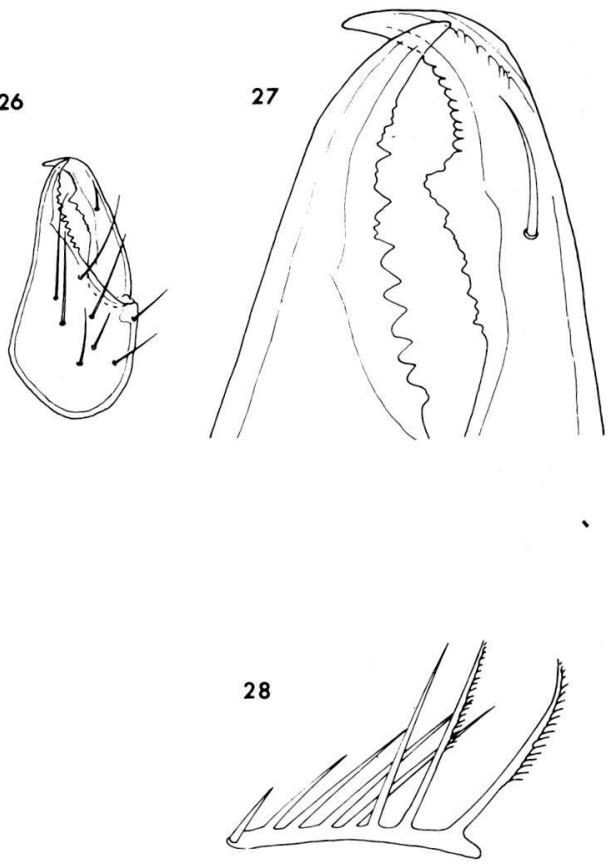

Fig. 23-28. Neobisium (Blothrus) karamani (Hadži) 1929.

(23) carapace, mâle $\mathrm{N}^{\mathrm{O}} 2 ;(24)$ épistome, mâle $\mathrm{N}^{\mathrm{O}} 2 ;$; 25 ) épistome, mâle $\mathrm{N}^{\mathrm{O}} 1 ;(26)$ chélicère, mâle $\mathrm{N}^{\mathrm{O}} 2 ;(27)$ doigts de la chélicère, mâle $\mathrm{N}^{\mathrm{O}} 2 ;(28)$ flagelle, mâle $\mathrm{N}^{\mathrm{O}} 2$. Echelle en $\mathrm{mm}$.

courtes. Le doigt fixe porte de 148 à 156 dents (chez la femelle, ce doigt porte 125 dents); elles sont asymétriques et à peu près de même taille. Dans leur partie terminale, ces dents portent des cupules d'émail; dans le quart proximal de la lame, les dents sont basses, plus larges que longues, et les quelques dernières dents n'ont pas de sommets émaillés. La lame du doigt mobile porte de 112 à 114 dents basses et obtuses, plus larges que longues dans la moitié distale du doigt; à partir du niveau de la trichobothrie ST, les dents sont asymétriques et leurs pointes dirigées en arrière. La lame de ce doigt se termine par des dents tout à fait basses et obtuses. Le doigt mobile porte 4 trichobothries, le doigt fixe 8 .

PATTES AMBULATOIRES - A l'avant et latéralement, le bord de la coxa I est étiré en un processus chitineux conique. Médialement, le bord de la coxa I forme quelques très petites pointes. La coxa II porte, antéro-latéralement, une bosse arrondie, de couleur chatain sombre. Les hanches des pattes marcheuses sont munies d'un plus grand nombre de soies que ne le sont les coxas de la femelle. Ainsi, la coxa I porte 7 soies (5-6 chez la femelle), la coxa II porte 7 ou 8 soies (5-7 
chez la femelle), la coxa III porte 6 soies ( 4 chez la femelle), et la coxa IV porte de 10 à 12 soies ( 8 chez la femelle).

DIMENSIONS (en $\mathrm{mm}$ ) - v. Tableaux $3 \& 4$.

MATERIAU - Deux mâles.

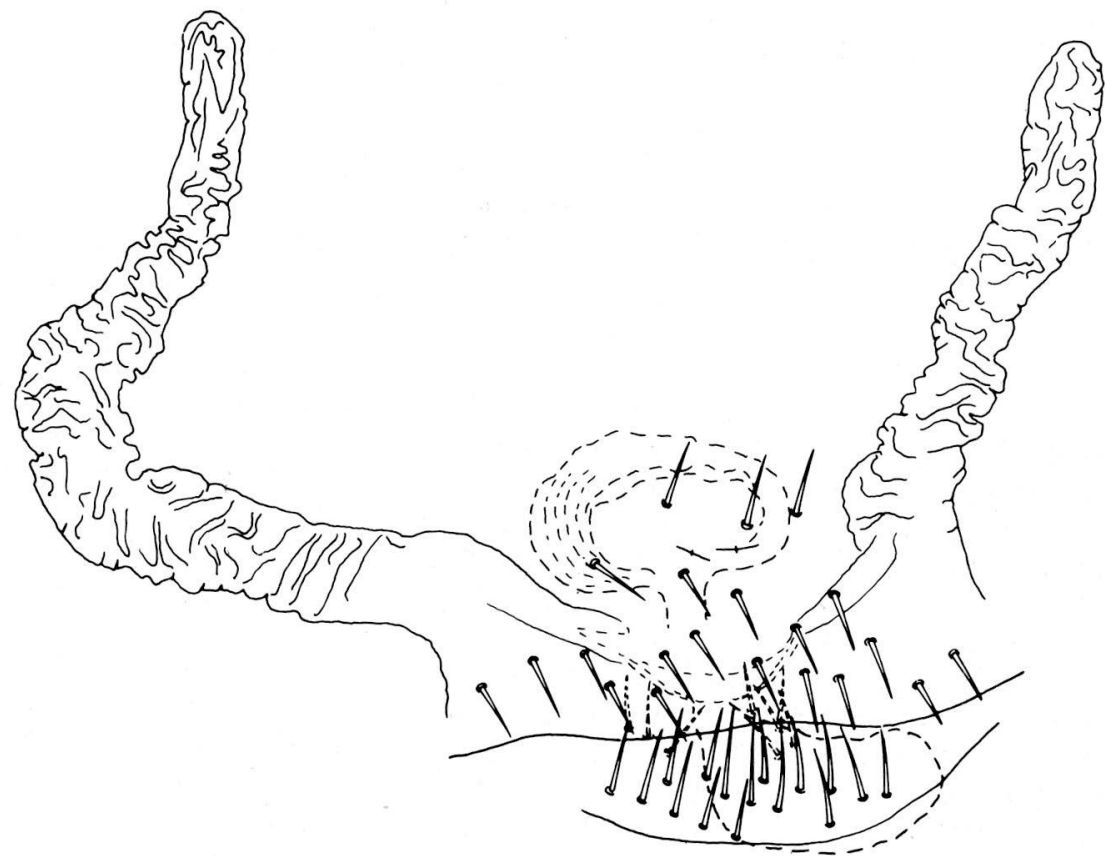

Fig. 29. Neobisium (Blothrus) karamani (Hadži) 1929, mâle $\mathrm{N}^{\circ}$ 2. région génitale; échelle en $\mathrm{mm}$. 


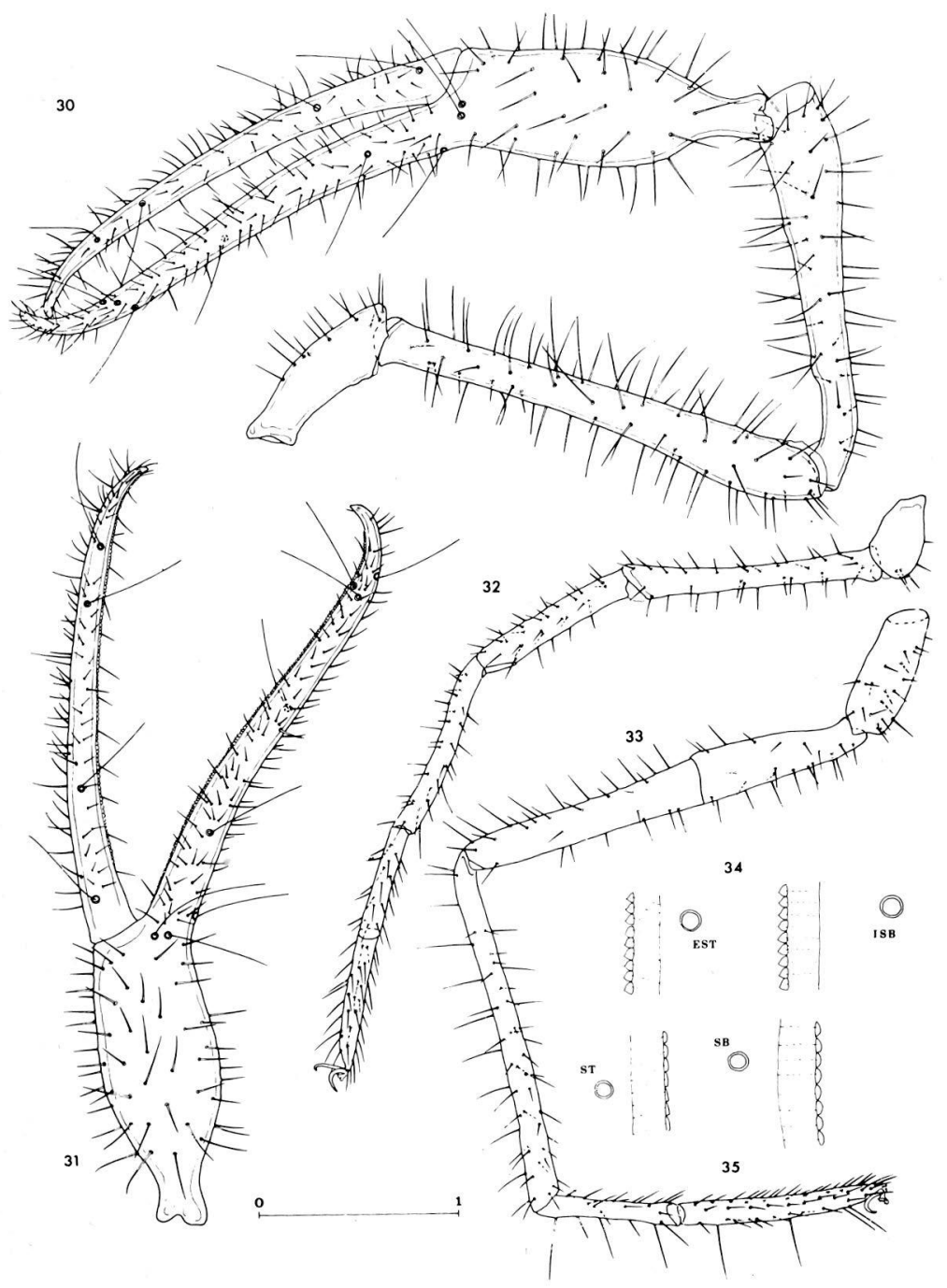

Fig. 30-35. Neobisium (Blothrus) karamani (Hadži) 1929., mâle N ${ }^{\mathrm{O}} 2$.

(30) patte-mâchoire; (31) pince; (32) patte ambulatoire II; (33) patte ambulatoire IV; (34) dents du doigt fixe de la pince (au niveau des trichobothries EST et ISB); (35) dents du doigt mobile de la pince (au niveau des trichobothries ST et SB). Echelle en $\mathrm{mm}$. 
Tableau 3. Les dimensions (en $\mathrm{mm}$ ) et les rapports morphométriques des structures différentes chez le Neobisium (B.) karamani (Hadži) 1929.

caractère

\section{Corps}

1. longueur

Céphalothorax

2. longueur

3. longueur du bord antérieur

4. largeur maximale

5. rapport: $2 / 4$

6. longueur du bord postérieur

7. rapport: $3 / 6$

Abdomen

8. longueur

9. largeur

Chélicères

10. longueur

11. largeur

12. épaisseur

13. longueur du doigt mobile

14. rapport: $10 / 13$

Pattes-mâchoires

15. longueur (avec la hanche)

16. longueur (sans la hanche)

17. rapport: $15 / 1$

18. longueur de la hanche

19. longueur du trochanter

20. largeur du trochanter

21. longueur du fémur

22. largeur minimale du fémur

23. largeur maximale du fémur

24. rapport: $21 / 23$

25 . longueur du tibia

26. largeur du tibia

27. rapport: $25 / 26$

28. longueur de la pince

29. largeur de la pince

30. rapport: $28 / 29$

31 . longueur de la main

32. rapport: $31 / 29$

33. longueur du doigt mobile

34. longueur du doigt fixe

35. rapport: $34 / 31$

36. distance de la base du doigt à B

37. distance de $\mathrm{B}$ à $\mathrm{SB}$

38. distance de SB à ST

39. distance de ST à T

40. distance de $\mathrm{T}$ au sommet du doigt mâle

(d'après nos données)

$$
3.23-3.41
$$

$1.15-1.17$

$0.84-0.89$

$0.93-0.94$

$1.22-1.26$

$0.84-0.85$

$1.00-1.05$

$2.00-2.26$

$0.905-0.91$

$0.42-0.425$

$0.35-0.36$

$0.57-0.58$

$1.57-1.59$

femelle

(d'après Hadži, 1929)

1.28

0.85

0.98

1.36

0.85

1.00

2.40

10.40

$9.42-9.43$

$3.05-3.22$

$0.97-0.98$

$0.95-0.96$

$0.32-0.34$

$2.37-2.39$

0.93

0.42

$-$

0.57

2.21

$0.18-0.185$

9.65

9.00

2.62

0.65

0.91

0.40

2.35

$0.31-0.33$

0.18

$7.24-7.645$

0.25

$2.02-2.06$

9.40

$0.38-0.39$

2.00

$5.28-5.32$

0.38

$4.02-4.08$

5.26

$0.56-0.59$

3.74

$6.915-7.18$

0.70

$1.54-1.59$

5.34

$2.61-2.84$

1.50

$2.36-2.49$

2.14

$2.43-2.54$

2.10

$1.52-1.65$

2.24

$0.22-0.25$

$0.52-0.56$

$0.91-0.93$

$0.26-0.30$

$0.42-0.44$ 
Tableau 4. Les dimensions (en $\mathrm{mm}$ ) et les rapports morphométriques des structures différentes chez le Neobisium (B.) karamani (Hadži) 1929.

caractère

mâle

(d'après nos données)

femelle (d'après Hadži,

Patte ambulatoire II

1. longueur (avec la hanche)

$5.345-5.39$ 1929)

2. longueur (sans la hanche)

$4.935-4.97$

4.96

3. longueur de la hanche

4. largeur de la hanche

5. longueur du trochanter

6. largeur du trochanter

7. longueur du fémur I

8. largeur du fémur I

9. longueur du fémur II

10. largeur du fémur II

11. longueur du tibia

12. largeur du tibia

13. longueur du basitarse

14. largeur du basitarse

15. longueur du télotarse

16. largeur du télotarse

Patte ambulatoire IV

17. longueur (avec la hanche)

18. longueur (sans la hanche)

19. longueur de la hanche

20. largeur de la hanche

21. longueur du trochanter

$0.41-0.42$

4.61

$0.43-0.45$

0.35

$0.25-0.26$

$1.30-1.32$

$0.185-0.19$

$0.84-0.86$

0.40

22. largeur du trochanter

$1.01+1.0 .16$

$0.11-0.12$

$0.58-0.60$

0.11

$0.75-0.77$

$0.10-0.11$

$7.18-7.25$

$6.55-6.63$

$0.62-0.63$

$0.37-0.38$

$-$

1.18

$-$

23. longueur du fémur 0.73

$0.25-0.27$

$2.08-2.12$

$0.25-0.27$

$1.95-1.96$

0.85

24. largeur du fémur

25. longueur du tibia

26. largeur du tibia

27. longueur du basitarse

28. largeur du basitarse

29. longueur du télotarse

30. largeur du télotarse

$\begin{array}{ll}0.16 & - \\ 0.74-0.75 & 0.74 \\ 0.13 & - \\ 1.05-1.07 & 0.80 \\ 0.13 & -\end{array}$

Les relations du Neobisium (Blothrus) karamani avec les autres espèces du genre

Entre les espèces $N$. (B.) princeps et $N$. (B.) karamani de nombreuses distinctions ont été constatées et qui sont exposées à la page 202 de ce travail. L'autre espèce des grottes de la Macédoine, le $N$. (B.) ohridanum presente aussi, par rapport au $N$. (B.) karamani, des différences nettes, constatées avec précision par Hadži (1940). D'autre part, les rapports de cette espèce avec le $N$. (B.) stankovici, troglobie de la Serbie orientale, ont été analysés récemment par Ćurčić (1972). 
En établissant les relations de parenté du $N$. (B.) karamani avec les autres espèces, Hadži (1929) a rapproché cette espèce de certains Pseudoscorpions blothroïdes des Karpates méridionales. Selon cet auteur, l'espèce $N$. (B.) brevipes (Frivaldsky) 1866, provenant des localités souterraines des régions de Turda et de Bihar dans les Alpes de Transylvanie se rapprocherait de l'espèce karamani. Si l'on compare les descriptions de cette espèce (Tömösváry 1882, Beier 1940) et de l'espèce karamani (Hadži 1929), on constate que les espèces karamani et brevipes diffèrent, entre autres, par la chétotaxie de la carapace et par la chétotaxie des tergites abdominaux. Des différences se manifestent également dans le nombre, dans la forme et dans la disposition des dents des chélicères, dans la forme des doigts de la pince et dans quelques rapports morphométriques. En plus, chez le $N$. (B.) karamani, certaines parties du corps sont plus longues, le fémur est plus gracile, le tibia de la patte-mâchoire est plus élargi dans sa partie distale, et la main est plus ovale que chez le $N$. (B.) brevipes. La parenté de ces deux espèces se manifeste, toutefois, par la similitude des articles de la patte-mâchoire et, dans une certaine mesure, de la pince, ainsi que par la similitude de certains rapports morphométriques.

L'espèce $N$. (B.) karamani se différencie aussi nettement de l'espèce $N$. (B.) leruthi Beier 1931, provenant des grottes des régions de Turda et de Bihar; ces distinctions se manifestent, avant tout, par la chétotaxie du côté dorsal de l'abdomen (le nombre de soies est plus grand dans l'espèce karamani), par la dentition du doigt mobile des chélicères, par la forme du tibia de la patte-mâchoire (dans l'espèce karamani, le côté latéral externe du tibia est concave, dans l'espèce leruthi il est plan), par le nombre et par la disposition des soies longues sur le tibia de la patte-mâchoire (dans l'espèce karamani, les soies longues sont développées sur le côté latéral interne du tibia, tandis que le tibia de l'espèce leruthi ne porte qu'une seule soie longue sur son côté dorsal), et par la forme de la main de la pince (dans l'espèce macédonienne, la main est plus ovale que dans l'espèce roumaine). En outre, ces deux espèces diffèrent encore par la forme du bord médian de la coxa I (lequel, dans l'espèce karamani, est étiré en quelques très petites pointes, tandis que dans l'espèce leruthi, il est "sehr stark zahnförmig vorgezogen"), par la longueur absolue de certaines parties du corps et, aussi par quelques rapports morphométriques.

L'espèce karamani se rapproche, cependant, de l'espèce leruthi par toute une série de caractères morphologiques, à savoir: la longueur relative de la carapace, la chétotaxie du bord postérieur du céphalothorax, la dentition du doigt fixe de la chélicère et, dans une certaine mesure, celle des doigts de la pince, la forme du fémur et jusqu'à un certain point celle de la pince de la patte-mâchoire, ainsi que certains rapports morphométriques.

De toutes façons, du point de vue zoogéographique, il est également intéressant de constater que le $N$. (B.) karamani est le plus proche voisin de l'espèce $N$. (B.) remyi Beier 1939 peuplant les grottes de la Serbie occidentale. La similitude et, par suite, la parenté de ces deux espèces s'observent surtout dans les caractères suivants, à savoir: dans la présence de l'épistome, dans la forme du fémur, de la pince et, dans une certaine mesure, du tibia de la patte-mâchoire, dans la dentition de la pince et 
dans certains rapports morphométriques. Les différences entre ces deux espèces analysées se manifestent par un plus grand nombre de soies au côté dorsal de l'abdomen dans l'espèce karamani, par la dentition du doigt mobile de la chélicère (dans l'espèce karamani, ce doigt porte une dent médiane nettement développée, tandis qu'une telle dent est absente dans l'espèce remyi), par la présence d'un tubercule au trochanter de la patte-mâchoire (dans l'espèce remyi, un tel tubercule n'est pas développé), par la forme du tibia de la patte-mâchoire (dans l'espèce karamani, cet article est distalement plus élargi que dans l'espèce remyi), et par la forme du bord médian de la coxa I (dans l'espèce karamani, cette partie est arrondie, tandis que dans l'espèce remyi, elle est nettement saillante).

Les espèces karamani et remyi appartiennent, d'après Beier (1939) à la série des espèces dite dalmatinum, dont le centre de distribution se trouve dans la zone méditerranéenne de la partie occidentale de la Péninsule des Balkans. A notre avis et d'après l'analyse comparative des caractères taxonomiques, à cette série appartiennent aussi les espèces brevipes et leruthi. A en juger d'après la répartition actuelle de ces quatre especces, la forme ancestrale de la série des espèces dalmatinum aurait peuplé non seulement la partie occidentale de la Péninsule, comme l'affirme Beier (1939), mais aussi un vaste territoire de la région intérieure des anciennes terres des Balkans. Cependant, le fait qu'un des centres importants de la radiation de ce groupe d'espèces soit situé dans la région dinarique, c'est-à-dire dans la partie occidentale de la Péninsule des Balkans, - reste irréfutable.

D'après notre opinion, le $N$. (B.) karamani représente un élément de la faune des Pseudoscorpions de la Macédoine. En ce qui concerne son habitat préféré, cette espèce est un hôte exclusif des stations souterraines. Du point de vue biogéographique, cependant, le $N$. (B.) karamani peut être considéré comme un relicte de la faune tertiaire des Arachnides méditerranéens, dont la différentiation endémique s'est faite dans les conditions de l'évolution du relief karstique de la Macédoine.*

\section{RESUME}

La grotte Kalina Dupka, située dans la montagne Bistra en Macédoine occidentale, est habitée par un Pseudoscorpion cavernicole du genre Neobisium Chamberlin 1930. Ce Pseudoscorpion diffère nettement des autres membres du sous-genre Blothrus Schiødte 1849, et appartient à la nouvelle espèce: $N$. (B.) princeps, dont les principaux caractères sont donnés dans le présent travail. Les plus proches voisins de cette espèce sont le $N$. (B.) spelaeum (Schiodte) 1849 , et le $N$. (B.) stygium Beier 1931, provenant des grottes de la Slovénie et de la Croatie. D'après la

*) Les échantillons des Pseudoscorpions, dont les caracteres morphologiques ont été analysés dans ce travail, appartiennent à la collection de Pseudoscorpions du Professeur Jovan Hadži (Ljubljana). Cette collection m'a été confiée en vue d'une étude scientifique grâce à l'amabilité de Docteur Jože Bole (Ljubljana), et je l'en remercie très sincèrement.

Je profite de cette occasion pour souligner l'aide du Professeur Dušan Manaković (Skoplje) et du Professeur Josif Josifovski (Skoplje), qui m'ont fourni des renseignements sur les grottes Zmejovica et Kalina Dupka. 
dentition de la pince, le $N$. (B.) princeps peut être considéré comme l'élément le plus primitif de la série princeps-stygium-spelaeum. La nouvelle espèce est spécialisée à l'extrême au mode de vie cavernicole. Au point de vue biogéographique, elle appartient à la faune de Pseudoscorpions endémiques de la Macédoine.

D'après la trouvaille de Pseudoscorpions de la grotte Zmejovica (région montagneuse du Poreče) nous avons confirmé la présence de l'espèce $N$. (B.) karamani (Hadži) 1929 dans la Macédoine occidentale. L'analyse morphologique des spécimens mâles, provenant de la nouvelle localité, nous a permis de compléter la descriptions de cette espèce, établie auparavant d'après un seul exemplaire femelle. La comparaison de l'espèce de Hadži avec le $N$. (B.) remyi Beier 1939, provenant des grottes de la Serbie occidentale, et avec le $N$. (B.) brevipes (Frivaldsky) 1866, et le $N$. (B.) leruthi Beier 1931, provenant des grottes de la Turda et du Bihar dans les Karpates méridionales, conduit à la constatation que les Pseudoscorpions cités appartiennent à un groupe d'espèces très voisines. A en juger d'après la répartition actuelle de ces espèces, il est possible que la forme initiale de cette série ait peuplé une large région des anciennes terres balkaniques.

Quant à ses préférences d'habitat, le $N$. (B.) karamani est un hôte exclusif du milieu souterrain. Du point de vue biogéographique, il peut être considéré comme un relicte de la faune tertiaire de Pseudoscorpions méditerranéens, dont la différenciation endémique s'est faite dans les conditions de l'évolution du relief karstique des parties méridionales de la Péninsule des Balkans.

En conclusion, d'après les connaissances actuelles et d'après les résultats de ce travail, les grottes de la Macédoine sont habitées par trois espèces endémiques de Pseudoscorpions du genre Neobisium (Blothrus) à savoir: $N$. (B.) ohridanum Hadži 1940, N. (B.) karamani (Hadži) 1929, et le N. (B.) princeps Ćurčić 1974. A en juger d'après les éléments de la faune des Pseudoscorpions blothroïdes connus actuellement, il est très possible que la Macédoine représente un des centres d'origine et de génèse de la faune autochtone résiduelle d'âge tertiaire.

\section{REZIME}

Pećinu Kalina Dupka na planini Bistra u zapadnoj Makedoniji naseljava jedna kavernikolna pseudoskorpija iz roda Neobisium Chamberlin 1930; ona se jasno razlikuje od drugih pripadnika podroda Blothrus Schiødte 1849, i pripada novoj vrsti: $N$. (B.) princeps, čije su osnovne karakteristike iznete u ovome radu. Novoj vrsti stoje ponajbliže $N$. (B.) spelaeum (Schiфdte) 1849, i $N$. (B.) stygium Beier 1931 iz pećina Slovenije i Hrvatske. Na osnovu denticije palpusa, $N$. (B.) princeps se može smatrati najprimitivnijim elementom serije princeps-stygium-spelaeum. Nova vrsta je ekstremno specijalizovana na pećinski način zivota; biogeografski, ona pripada fauni endemičnih pseudoskorpija Makedonije.

$\mathrm{Na}$ osnovu uzorka pseudoskorpija iz pećine Zmejovica, koja pripada planinskoj oblasti Poreče, potvrdjeno je prisustvo vrste $N$. (B.) karamani (Hadži) 1929, u zapadnoj Makedoniji. Na osnovu morfološke analize mužjaka iz novog nalazista, dopunjen je opis ove vrste, inače zasnovane samo na jednom primerku ženke. 
Uporedjivanje Hadžijeve vrste sa $N$. (B.) remyi Beier 1939 iz pećina zapadne Srbije, i sa $N$. (B.) brevipes (Frivaldsky) 1866 i $N$. (B.) leruthi Beier 1931 iz pećina Turde i Bihara $u$ južnim Karpatima ukazuje na konstataciju da navedene pseudoskorpije pripadaju grupi blisko srodnih vrsta. Sudeći prema današnjem rasprostranjenju vrsta, predačka forma ove serije mogla je naseljavati široko područje starog balkanskog kopna.

Biotopski, N. (B.) karamani je izraziti stanovnik podzemnih staništa. Sa biogeografskog gledišta, on se može smatrati reliktom tercijarne faune mediteranskih pseudoskorpija, čija se endemična diferencijacija odigravala u uslovima evolucije kraškog reljefa južnih delova Balkanskog poluostrva.

U pećinama Makedonije, prema dosadašnjem poznavanju i prema rezultatima ovoga rada, žive tri endemične pseudoskorpije iz roda Neobisium (Blothrus). To su: N. (B.) ohridanum Hadži 1940, N. (B.) karamani (Hadži) 1929 i N. (B.) princeps Ćurčić 1974. Sudeći po do sada poznatim elementima faune blotroidnih pseudoskorpija, sasvim je moguće da Makedonija, kao deo starog balkanskog kopna, predstavlja jedan od centara nastanka i geneze autohtone reliktne faune tercijarne starosti.

\section{BIBLIOGRAPHIE}

BEIER, M. 1928. Die Pseudoskorpione des Wiener Naturhistorischen Museums. I. Hemictenodactyli. Ann. Naturhistor. Mus. Wien, 42: 285-324.

BEIER, M. 1939. Die Höhlenpseudoscorpione der Balkanhalbinsel. Stud. allg. Karstforsch. Brünn, Biol. Ser., 4: 1-83.

BEIER, M. 1939a. Etudes biospéologiques. XV (1) 4. Pseudoscorpionidea de Roumanie. Bull. Mus. royal Hist. Nat. Belgique, 15 (39): 1-21.

BEIER, M. 1940. Zur Phylogenie der troglobionten Pseudoscorpione. VI Congr. Int. Entom. Madrid 1935: 519-527.

BEIER, M. 1963. Ordnung Pseudoscorpionidea (Afterskorpione). Bestimmungsbücher zur Bodenfauna Europas, 1: 1-313. Akademie-Verlag, Berlin.

BEIER, M. 1973. Beiträge zur Pseudoscorpionidenfauna Anatoliens. Fragm. Entomologica (Roma), 8 (5): 223-236.

ĆURČIĆ, B. P. M. 1972. Neobisium (Blothrus) stankovici, nouvelle espèce de Pseudoscorpions cavernicoles de la Serbie orientale. Fragm. Balcanica (Skopje), 9 (9): 85-96.

ĆURČIĆ, B. P. M. 1974. Pseudoscorpiones. In: Catalogus Faunae Jugoslaviae. Slov. Akad. Znan. Umetn. Ljubljana, 3 (4): 1-36.

HADŽI, J. 1929. Obisium (Blothrus) Karamani, Pseudoscorpion nouveau de la Serbie du Sud. Acta Soc. Entom. Iugosl. Beograd, 3-4 (1-2): 1-11.

HADŽI, J. 1940. Eine neue Art von Höhlen-Pseudoskorpioniden aus Südserbien, Neobisium (Blothrus) ohridanum sp. n. Bull. Soc. Sc. Skopje., Sect. Sc. Nat., 8: 129-135.

HELVERSEN, O. v. \& J. MARTENS 1972. Unrichtige Fundort-Angaben in der ArachnidenSammlung Roewer. Senckenbergiana biol., Frankfurt, 53 (1-2): 109-123.

JOSIFOVSKI, J. 1973. Communication personelle.

JOSIFOVSKI, J. manuscrit. Karst i karsne pojave na planini Bistri.

JOVANOVIĆ, P. S. 1928. Karsne pojave u Poreču (Phénomènes karstiques dans le Poreče). Bull. Soc. Sc. Skopje, Sect. Sc. Nat., 4 (1): 1-46.

MAHNERT, V. 1971. Neobisium (Blothrus) kwartirnikovi nov. spec. (Pseudoscorpionidea) aus Bulgarien. Arch. Sc. Genève, 24 (3): 383-389.

MULLER, J. 1931. Nuovi Pseudoscorpioni cavernicoli appartenenti al sottogenere Blothrus Schiodte. Bull. Soc. Entom. Ital., 63: 125-127.

TOMOSVARY, O. 1882. Pseudoscorpiones faunae hungaricae (A magyar fauna álskorpiói). Magyar Akad. Math. Termész. Közlem., 18: 135-256. 